\title{
Necrotizing Fasciitis Associated with Pregnancy: a Population-Based Cohort Study
}

\author{
Lavi Oud $\cdot$ Phillip Watkins
}

To view enhanced content go to www.infectiousdiseases-open.com

Received: June 23, 2014 / Published online: July 29, 2014

(c) The Author(s) 2014. This article is published with open access at Springerlink.com

\section{ABSTRACT}

Introduction: Necrotizing fasciitis (NF) is a rare complication in pregnant women. There have been no population-level data reported to date on its epidemiology, clinical features, resource utilization, and outcomes.

Methods: This was a retrospective, populationbased cohort study, using the Texas Inpatient Public Use Data File to identify pregnancyassociated hospitalizations for the years 2001-2010. Hospitalizations with a diagnosis of $\mathrm{NF}$ were then identified using the International Classification of Diseases, Ninth Revision, Clinical Modification code 728.86. Denominator data for incidence estimates were derived from the Texas Center for Health

Electronic supplementary material The online version of this article (doi:10.1007/s40121-014-0031-0) contains supplementary material, which is available to authorized users.

L. Oud $(\square)$

Division of Pulmonary and Critical Care Medicine, Department of Internal Medicine, Texas Tech University Health Sciences Center at the Permian Basin, 701 W. 5th St., Odessa, TX 79763, USA

e-mail: lavi.oud@ttuhsc.edu

P. Watkins

Clinical Research Institute, Texas Tech University

HSC, Lubbock, USA
Statistics reports of live births, abortions and fetal deaths, and previously reported population-based, age-specific linkage data on miscarriage, and were used to estimate the annual total number of pregnancies (TEP). The incidence of pregnancy-associated NF (PANF), hospitalization type, clinical features, resource utilization and outcomes were examined.

Results: There were 4,060,201 pregnancyassociated hospitalizations and 148 PANF hospitalizations during study period. Postpartum hospitalizations accounted for 82.4\% of all PANF events, and intensive care unit care was required in $61.5 \%$. The key trends noted between 2001-2002 and 2009-2010 included rising incidence of PANF from 1.1 vs. 3.8 per 100,000 TEP-years $(P=0.0001)$, chronic comorbidities $0 \%$ vs. $31.7 \%(P=0.0777)$, and development of organ failure in $9.1 \%$ vs. $31.7 \%$ $(P=0.0302)$. There was no significant change in total hospital charges or hospital length of stay. Three patients (2\%) died in the hospital and $55 \%$ of survivors had routine home discharge.

Conclusions: The present cohort of PANF is the largest reported to date. The incidence of PANF rose nearly 3.5 -fold over the past decade, with most events developing following delivery 
hospitalization. Chronic illness has been increasingly present, along with rising severity of illness. The majority of patients required ICU care. Hospital mortality was lower than that reported for NF in the general population. The sources of the observed findings require further study.

Keywords: Mortality; Necrotizing fasciitis; Organ failure; Pregnancy; Resource utilization

\section{INTRODUCTION}

Pregnancy is associated with an increased risk of infection, in part due to various pregnancyrelated mechanical and physiological changes [1]. In addition, recent evidence suggests that pregnancy is associated with an immunological shift away from inflammatory processes and inflammatory cytokines and toward a more anti-inflammatory immunologic state [2]. These changes may also play a role in the maternal response to overwhelming infection and subsequent sepsis [2].

Despite improvements in medical care and preventive measures, infectious complications remain a major source of pregnancy-related mortality in both developing and developed countries worldwide [3], reported to be the 5th most common cause of maternal death [1]. A recent review conducted by the World Health Organization has estimated the global burden of maternal sepsis to be more than $6,900,000$ cases per year [4].

Necrotizing fasciitis (NF) is a soft tissue infection manifesting as necrosis of subcutaneous tissues and fascia. Although rare, NF commonly results in severe and often fatal illness with high resource utilization. Case fatality associated with NF has been reported to exceed $40 \%$ in single-center studies [5], while reports on larger cohorts described case fatality around $5-12 \%[6,7]$.

Pregnancy-associated NF (PANF) has been described in multiple reports. However, because of its rarity, descriptions of NF in the obstetric population to date were limited to case reports [8-10] or small case series [11, 12], and was absent in a population study of invasive streptococcal infections in the postpartum period [13]. Thus, the epidemiology of PANF is presently unknown, with limited data on its clinical characteristics, resource utilization and outcomes.

The aim of this first population-level study to date, to the authors' knowledge, was to examine the epidemiological, clinical, resource utilization, outcome characteristics, and secular trends of pregnancy-associated NF.

\section{MATERIALS AND METHODS}

\section{Data Sources}

Data were obtained from the Texas Inpatient Public Use Data File (TIPUDF), a longitudinal data set maintained by the Texas Department of State Health Services [14]. The data set includes detailed de-identified inpatient discharge data from all state-licensed hospitals, with the exception of those exempt by state statute from reporting to the Texas Health Care Information Collection. Exempt hospitals include (a) those that do not seek insurance payment or government reimbursement and (b) Selected rural providers, based on bed number and local county population [14]. The facilities included in the mandated report account for $93-97 \%$ of all hospital discharges. The TIPUDF data set includes demographic, clinical, resource utilization, and outcome information. The data set includes up to 25 
discharge diagnoses, and up to 25 procedures, coded using the International Classification of Diseases, Ninth Revision, Clinical Modification (ICD-9-CM).

Data on the annual number of pregnancies, live births, abortions, fetal deaths, and their related demographic characteristics were obtained from the Vital Statistics Annual Reports, compiled by the Center for Health Statistics at the Texas Department of State Health Services [15].

The TIPUDF is a publicly available, deidentified data set, and therefore this study was determined to be exempt from formal review by the Texas Tech Health Sciences Center Institutional Review Board. This article does not involve any new studies with human or animal subjects performed by any of the authors.

\section{Study Population}

Texas residents with pregnancy-related hospitalizations between 2001 and 2010 were identified using ICD-9-CM codes (Supplemental Appendix 1). Subsequently, an ICD-9-CM code 728.86 was used to identify patients with a primary or secondary diagnosis of NF.

\section{Data Collection}

Data were collected on patients' age, race (categorized as non-Hispanic black [black], non-Hispanic white [white], Hispanic, and other), health insurance (categorized as private, Medicaid, uninsured, and other), chronic comorbid conditions (based on the Deyo-Charlson index [16]), obesity, smoking, drug and alcohol abuse, other sites of infection (Supplementary Appendix 2), reported microorganisms (Supplementary Appendix 3), type and number of failing organs
(Supplementary Appendix 4), admission to an intensive care unit (ICU), life-support interventions (mechanical ventilation, central venous catheterization, hemodialysis, and tracheostomy) (Supplementary Appendix 5), total hospital charges, hospital length of stay, and disposition at the end of hospitalization. Severity of illness was based on the number of failing/dysfunctional organs (organ failure $[\mathrm{OF}]$ ), as modeled by the coding system reported by Lagu et al. [17].

Type of pregnancy-associated hospitalizations were categorized into the following mutually exclusive, hierarchical groups, using pregnancy-associated ICD-9-CM codes: (a) fetal loss (pregnancies with abortive outcome, excluding induced abortion), (b) induced abortion (c), delivery (based on the approach described by Kuklina and colleagues [18]), (d) postpartum (hospitalizations with a an ICD-9-CM code for puerperal complications, without pregnancyrelated diagnosis codes of groups $\mathrm{a}-\mathrm{c}$ ), and (e) antepartum (hospitalization with pregnancy-related diagnosis, but without pregnancy-related diagnosis codes of groups a-d).

\section{Outcomes}

The primary outcome was hospital mortality. Secondary outcomes included number and type of $\mathrm{OF}$, resource utilization, and disposition among hospital survivors.

\section{Data Analysis}

To derive the incidence of PANF events across the full spectrum of pregnancy outcomes, the number of annual total estimated pregnancies (TEP) was calculated. TEP was a combination of the number of live births, fetal deaths (events 
reported by the state, occurring at $\geq 20$ weeks of gestation), induced abortions, and estimates of the annual number of fetal losses (events occurring at $<20$ weeks of gestation, including miscarriage, ectopic and molar pregnancies). The estimation of the annual number of fetal losses was based on the study by Nybo Anderson et al. [19]. This was a population-based linkage study of the association of maternal age with fetal loss, reporting rates of fetal loss for pregnancies intended to be carried to term, thus adjusting for overestimates resulting from fetal loss events prior to planned abortion. The reported number of live births and fetal deaths was used to derive the number of fetal losses. Because TIPUDF provides discharge-level, rather than patient-level information, PANF events were reported as number of hospitalizations. Incidence rates of patients' hospitalizations with a diagnosis of PANF per 100,000 TEP were calculated. Direct age adjustment using 5-year age strata was performed. Two PANF hospitalizations associated with fetal loss/ induced abortion could not be adequately classified to only one group (that is, either fetal loss or induced abortion), because their only pregnancy-associated ICD-9-CM code was 639.XX (complications following abortion and ectopic and molar pregnancies). A "worst-case" upper incidence estimate (reported parenthetically) was recalculated for both fetal loss and induced abortion PANF hospitalizations, assuming alternately that the unclassified hospitalizations were only fetal loss- or only induced abortion related.

Multiple sensitivity analyses were performed to examine the robustness of the incidence estimates. Although TIPUDF is reported to include $93-97 \%$ of annual hospital discharges, the annual incidence of PANF was reanalyzed, assuming the data set captures only $90 \%$ of all hospital discharges. In addition, because the non-reporting hospitals are skewed toward rural facilities, potentially affecting care patterns, we assumed the incidence of PANF is higher, up to $50 \%$ above that for reporting hospitals.

In addition, the incidence of PANF was reanalyzed, assuming that the rate of fetal loss among Texas residents is twice as high as the $13.5 \%$ rate reported by Nybo et al. [19]. This higher rate exceeds the upper estimated rate of fetal loss of $22 \%$ reported in a recent systematic review by Ammon Avalos et al. [20].

The mortality associated with PANF was examined as case fatality (defined as the number of PANF hospitalizations who died in the hospital divided by the total number of PANF hospitalizations for an examined group).

Group data were reported as numbers (percentages) for categorical variables and mean (standard deviation [SD]) or median (interquartile range [IQR]) for continuous variables, as appropriate. Ninety-five percent confidence intervals $(95 \% \mathrm{CI})$ were calculated. Distribution of normality was examined by Kolmogorov-Smirnov test. Categorical data were compared by 2 -sided $X^{2}$. Mann-Whitney $U$ test and $t$ test were used to compare continuous data, as appropriate. A regression analysis was used to explore the annual trends of PANF incidence. When examining trends of key characteristics at the start vs. end of past decade combined 2-year data were used to enhance precision of comparisons. Total hospital charges were examined using inflation-adjusted (2010) dollars. All statistical analyses were performed using MedCalc version 12.7.0 (MedCalc Software, Ostend, Belgium) and SAS version 9.3 (SAS Institute, Cary, NC, USA). A 2 -sided $P$ value $<0.05$ was considered statistically significant. 


\section{RESULTS}

There were 4,060,201 pregnancy-associated hospitalizations and 148 PANF hospitalizations, with 5,347,084 total estimated pregnancies during the 2001-2010 period. The characteristics of PANF hospitalizations are detailed in Table 1. Hispanic women constituted the largest group (42.6\%) of PANF hospitalizations, reflecting the obstetric population in Texas. Medicaid was the most common type of health insurance (51.4\%). Only a minority of women (17.6\%) had reported chronic comorbid conditions, with diabetes mellitus noted in $50 \%$ of the latter. Drug and tobacco abuse were rare. Obesity was reported in $22.3 \%$ of PANF hospitalizations. Postpartum hospitalizations accounted for $82.4 \%$ of all NF events, while NF hospitalizations associated with miscarriage or abortion were rare. The incidence of PANF hospitalizations rose by $14 \%$ per year.

Other (non-NF) sites of infection were reported in 40 (27\%) PANF hospitalizations. The most common reported sites included the genital tract $(14.2 \%)$, respiratory, and intra-abdominal infections $(6.8 \%$ each). Microbiology data were reported in 61 (41.2\%) hospitalizations. When analysis was restricted to PANF hospitalizations with reported microbiology data, who had no other reported sites of infection $(n=52)$, single bacteria was predominant, noted in $65.4 \%$, being polymicrobial in the remainder. The following microbial isolates are restricted to these 52 PANF hospitalizations. Gram-positive bacteria were reported in 90.4\%, Gram-negative bacteria in $36.5 \%$, and anaerobes in $2 \%$. No fungi were reported. Among hospitalizations with reported Gram-positive bacteria, streptococcal species were reported in $55.3 \%$ and staphylococcal
Table 1 Characteristics of hospitalizations with pregnancy-associated necrotizing fasciitis

\begin{tabular}{|c|c|}
\hline Characteristic & $n=148$ \\
\hline \multicolumn{2}{|l|}{ Age (years, $n[\%]$ ) } \\
\hline$<20$ & $14(9.5)$ \\
\hline $20-34$ & $110(74.3)$ \\
\hline$\geq 35$ & $24(16.2)$ \\
\hline \multicolumn{2}{|l|}{ Race, $n(\%)$} \\
\hline Hispanic & $63(42.6)$ \\
\hline White & $53(35.8)$ \\
\hline Black & $22(14.9)$ \\
\hline Other & $10(6.8)$ \\
\hline \multicolumn{2}{|l|}{ Health insurance, $n(\%)$} \\
\hline Private & $57(38.5)$ \\
\hline Medicaid & $76(51.4)$ \\
\hline Uninsured & $11(7.4)$ \\
\hline Other & $4(2.7)$ \\
\hline \multicolumn{2}{|l|}{ Chronic comorbidities, $n(\%)^{\mathrm{a}}$} \\
\hline Any & $26(17.6)$ \\
\hline Diabetes mellitus & $13(8.8)$ \\
\hline Chronic pulmonary disease & $4(2.7)$ \\
\hline Chronic kidney disease & $3(2.0)$ \\
\hline Deyo-Charlson score (mean $[\mathrm{SD}]$ ) & $0.27(0.69)$ \\
\hline \multicolumn{2}{|l|}{ Other conditions, $n(\%)^{\mathrm{b}}$} \\
\hline Smoking & $5(3.4)$ \\
\hline Drug abuse & $3(2.0)$ \\
\hline Alcohol abuse & $0(0)$ \\
\hline Obesity & $33(22.3)$ \\
\hline \multicolumn{2}{|c|}{ Type of pregnancy-related hospitalization, $n$ (\%) } \\
\hline Fetal loss ${ }^{c}$ & $1(0.7) / 2(1.4)$ \\
\hline Abortion $^{c}$ & $0(0) / 1(0.7)$ \\
\hline Antepartum & $8(5.4)$ \\
\hline Delivery & $16(10.8)$ \\
\hline Postpartum & $122(82.4)$ \\
\hline
\end{tabular}

$n$, Number of patients; ICD-9-CM, International Classification of Diseases, Ninth Revision, Clinical Modification; SD, standard deviation

a Based on conditions included in the Deyo-Charlson comorbidity index

b Refers to comorbid conditions not included in the DeyoCharlson index

c There was 1 miscarriage/abortion-related hospitalization whose only pregnancy-related diagnosis was ICD-9-CM code 639.XX, precluding separation to one group; upper estimates of the number and percent of fetal loss hospitalizations were provided after the slash for each 
species in 51.1\%. Group A streptococci were reported in $6(11.5 \%)$ PANF hospitalizations without non-NF infection site. Among hospitalizations with reported Gram-negative bacteria, Proteus species (52.6\%) and E. Coli (36.8\%) were the most common isolates.

The key changes of epidemiological, clinical, and resource utilization features among PANF hospitalizations between 2001-2002 and 2009-2010 are outlined in Table 2. The incidence of PANF hospitalizations rose from 1.1 to 3.8 per 100,000 TEP-years $(P=0.0001)$, and was most common among black women. Estimates of the annual incidence of PANF remained unchanged on reanalyzing the data, assuming increased rates of fetal loss ( $P$ values ranging from 0.6612 to 0.8319 ) or with lower rate of statewide reported hospitalization, coupled with higher rates of PANF in unreported hospitalizations ( $P$ values ranging from 0.5637 to 0.7815 ). No significant change was noted among women aged 35 years or older $(P=0.2638)$. Chronic comorbidities were reported in nearly a third of PANF hospitalizations at the end of last decade, while none were reported in 2001-2002. The rate of reported obesity rose more than threefold, though the change did not reach statistical significance. One or more OFs were reported in 43 (29.1\%) PANF hospitalizations, rising from $9.1 \%$ to $31.7 \%$ during past decade $(P=0.0302)$. OF was reported nearly twice as commonly among PANF hospitalizations with chronic comorbidities, as compared to those without $(46.2 \%$ vs. $24.6 \% ; P=0.0483)$. Use of life-support interventions rose during study period, but did not reach statistical significance.

Admission to an ICU was required in $61.5 \%$ of PANF hospitalizations. Though downtrending over the past decade, there was no significant change in hospital length of stay $(P=0.4863)$ and total hospital charges
$(P=0.3642)$ among PANF patients. The average inflation-adjusted (2010 dollars) total hospital charges per PANF hospitalization were $\$ 102,434$.

Three (2\%) patients died during hospitalization. Among survivors, 80 (55\%) had routine home discharge, 50 (34\%) required home health care, and 14 (10\%) were discharged to another facility. No change was found in transfers to other institutions over the past decade (data not shown).

\section{DISCUSSION}

The incidence of PANF hospitalizations has risen nearly 3.5-fold over the past decade. PANF was infrequently associated with chronic comorbidity, while showing increased severity of illness over time. Most women with PANF in our cohort required admission to an ICU, with a trend of increasing use of life-support interventions. PANF required prolonged hospitalization and high hospital charges. Case fatality was low in the present cohort. However, hospital survivors sustained persistent morbidity with only about half having a routine home discharge.

The present study is, to the authors' knowledge, the first population-level examination of PANF, reflecting the rarity of this complication in obstetric patients. These findings of rising PANF incidence from about 1 to nearly 4 hospitalizations per 100,000 TEP cannot be directly compared with reports of NF in the general position, in part due to differences in age, prevalence of chronic comorbid conditions and preceding clinical interventions. A commonly cited multistate incidence estimate of NF in the US is 4 per 100,000 , based on the report by Ellis Simonsen et al. [21] using administrative data from 1997 to 2002. Because the incidence of NF rises with 
Table 2 Key changes of epidemiology, patient characteristics, resource utilization and outcomes of hospitalizations with pregnancy-associated necrotizing fasciitis

\begin{tabular}{llll}
\hline Variable & $\mathbf{2 0 0 1 - 2 0 0 2}(\boldsymbol{n}=\mathbf{1 1})$ & $\mathbf{2 0 0 9 - 2 0 1 0}(\boldsymbol{n}=\mathbf{4 1})$ & $\boldsymbol{p}$ \\
\hline Age-adjusted incidence (per 10 & TEP-years) & & \\
All & 1.1 & 3.8 & 0.0001 \\
Hispanic & 0.6 & 3.5 & 0.0023 \\
White & 1.6 & 4.2 & 0.0396 \\
Black & 0.8 & 4.9 & 0.0498 \\
Age $\geq 35$ years (\%) & 63.6 & 39 & 0.2638 \\
Chronic comorbidity (\%) & 31.7 & 0.0777 \\
Obesity (\%) & 0 & 29.3 & 0.3271 \\
$\geq 1$ Organ failures (\%) & 9.1 & 31.7 & 0.0302 \\
Procedures & 9.1 & & 0.2077 \\
Mechanical ventilation, (\%) & & 22 & 0.8027 \\
Central venous catheterization (\%) & 36.4 & 46.3 & 0.4757 \\
Hemodialysis (\%) & 0 & 2.4 & \\
Hospital length of stay (days) & & & 0.3642 \\
Mean (SD) & $16.2[11.1]$ & $12.1[7.3]$ & \\
Median [IQR] & $12[6-27]$ & $11[8-13]$ & \\
Hospital charges & $88,216[65,982-133,314]$ & $71,161[51,497-119,577]$ & 0.4867 \\
(Dollars, median [IQR]) & & &
\end{tabular}

$T E P$ Total estimated pregnancies, $I Q R$ interquartile range, $n$ number of patients, $S D$ standard deviation

${ }^{a}$ Chronic comorbidities from the Deyo-Charlson index

age $[6,22]$, it may be hypothesized that at the end of last decade the incidence of PANF in this cohort may have exceeded same-age development of NF in the general population. Markedly, lower incidence of NF was found by Mulla et al. [23] in another population study, using similar approach, with NF reported in 1.3 per 100,000 hospital discharges in Florida in 2001. However, the investigators focused only on NF as primary diagnosis. There are no more recent population-level data on the incidence of NF in the United States (US). Further studies are needed to corroborate our findings.
Several possible explanations should be considered for the apparent rise of incidence of PANF in this cohort. These findings may reflect increasing diagnosis of less severe skin and soft tissue infections as NF over time. However, this explanation is not supported by the evidence of rising severity of illness, matched by trends in use of life-support interventions, and unchanged resource utilization.

Increased clinician awareness of a specific clinical condition should be considered as an alternative source of an apparent rise in its 
incidence. However, this explanation is implausible in the case of PANF, as it remains a very rare complication, as evidenced in the current study with NF codes used in $0.004 \%$ of pregnancy-associated hospitalizations, and with most clinicians and hospitals in the state never encountering a PANF patient. It may thus be hypothesized that the present findings reflect actual rise in the incidence of PANF in the state. There are several possible explanations for rising incidence of PANF in this cohort. Chronic comorbidities, well known to increase risk of infection and NF [24] were present in nearly one-third of PANF hospitalizations at the end of study period. In addition, obesity was increasingly present in our cohort. Obesity is a well-known risk factor for NF [6], has been associated with increased risk of infections in pregnancy [25], and is more specifically linked with increasing risk of cesarean section $[25,26]$. The latter has been often associated with PANF in prior reports $[11,12]$. It is likely that the rate of obesity was underreported in this cohort, as can be the case in administrative data sets [27]. The rising rate of cesarean section in the US over the past decade [28] may have contributed to the rising incidence of PANF, a hypothesis supported by our findings of the majority of reported NF events occurring as postpartum hospitalizations. However, the de-identified structure of the administrative data set used in the present study precludes linking postpartum hospitalizations to specific preceding delivery hospitalizations to confirm this hypothesis. Additional study in other states and nationally is required to further elucidate the epidemiology of PANF.

Findings of the race/ethnicity composition of the women in the present study and the predominance of Medicaid as the most common type of health insurance, reflect the obstetric population in Texas, but may vary in other settings. The age distribution noted in the present cohort is in line with the majority of pregnancies occurring in the 20-34 years age group.

The majority of PANF hospitalizations did not have reported chronic comorbidities. This finding contrasts reports on NF in the general population, with the majority of patients having one or more chronic illnesses [6]. However, when chronic comorbidities were present in patients, diabetes was the predominant one, similar to reports in the general population with NF [6, 7]. These results are in agreement with reported cases and case series of PANF, with most affected patients without chronic illness. Obesity was reported in about 1 in 5 of our patients in this study and, as noted earlier, may have been underreported. On the other hand, obesity was prevalent in prior case series of PANF, reported in $67 \%$ [11] and $83 \%$ [12] of their patients.

The present study revealed that the majority of PANF events were during the postpartum period and during separate hospitalization after delivery, while miscarriage and abortion were rarely associated with this complication. These findings extend prior case series and case reports describing PANF events mostly in the postpartum period, often occurring following hospital discharge $[11,29,30]$. Moreover, the time to development of PANF, while rapid at times [31] can be prolonged. In a case series of PANF following cesarean section by Goepfert et al. [11], the authors reported the time difference from the procedure to diagnosis of NF ranging from 5 to 17 days and exceeding 1 week in 6/9 patients. These findings may explain the prevalent need for readmission following delivery hospitalization noted in the present cohort. However, as noted above, events prior to postpartum hospitalization cannot be ascertained and sequence of events 
(i.e., a surgical procedure preceding or following NF) cannot be inferred in administrative data sets. Thus, it can only be hypothesized that preceding procedures (i.e., cesarean section, episiotomy) may have contributed to postpartum NF events.

An additional site of infection was reported in about 1 in 4 PANF hospitalizations, mostly involving the genital and respiratory tracts. It is unclear whether reported infections preceded, followed, or occurred synchronously with PANF. Previous reports noted additional infections in some PANF patients. Goepfert et al. [11] reported an additional site of infection in $5 / 9$ of their patients, with chorioamnionitis and endometritis accounting for most. Appendicitis was reported by Penninga et al. [9] and in one of three PANF cases by Schumacher et al. [29]. Other sites of infection were reported in the general population with NF in 30\% [32] to 76\% [33] of patients.

Microbiology data were not reported in 59\% of PANF hospitalizations. Similar degree of underreporting was noted by others in national data sets, with microbiology information absent in up to $65 \%$ [34]. There was a predominance of monomicrobial pathogens in this cohort, mostly streptococcal species with few reports of group A streptococci. In a recent report by Aronoff et al. [13] on postpartum invasive streptococcal infections among women hospitalized in the state of Florida, based on both required reporting on invasive streptococcal infections and hospital discharge data, the investigators did not identify any PANF events among the described 7 hospitalizations. These findings underscore the rarity of streptococcal PANF and are in accord with the very few reported group A streptococcal isolates in our cohort over a much longer study period in a much larger population. The findings in the present study are in contrast with the polymicrobial etiology of most events of NF in the general population [24]. However, these results may have been affected by underreporting of microbiology, analysis restricted to PANF hospitalizations without other reported sites of infection, and administrative data precluding information on specimen sources. Previous reports of PANF varied in microbiology findings. Single case reports often described monomicrobial infections [8-10, 29, 31], while case series tended to report polymicrobial NF $[11,12]$.

$\mathrm{NF}$ is commonly considered to be a critical illness, with reports in the general population often focused on patients managed in the ICU [32]. This study revealed that nearly $60 \%$ of PANF hospitalizations required ICU care. These findings, coupled with the relatively low frequency of $\mathrm{OF}$ in this cohort, suggest a broader spectrum of illness among women with PANF than has been previously described, likely reflecting focus on more severe illness in individual case reports. These findings are similar to those reported by Tillou and colleagues in the US, describing ICU admission in $61 \%$ of their patients with NF in the general population [35]. The latter results are also remarkably similar to reports on NF in the general population in Australia [33] and New Zealand [36], showing need for ICU care in 63\% and $56 \%$ of their patients, respectively. Nevertheless, critical care utilization patterns can vary across countries [37] and regionally [38], limiting a direct comparison. Indeed, focus only on ICU-managed NF can underestimate the burden of NF in the population.

The respiratory, circulatory and renal systems were the most commonly involved with $O F$ in the present study. Previous case series of PANF and studies in the general population with NF did not systematically 
describe patterns of OF [9-12, 29]. When selected OFs were systematically examined, investigators reported renal, circulatory, and respiratory systems as the most commonly affected in that order [39]. However, the investigators restricted their definition of respiratory failure to patients requiring invasive mechanical ventilation, thus likely underestimating the frequency of this complication and overall OF. In a recent report by Das et al. [36], focusing on selected $\mathrm{OF}$, shock and renal failure were each present in $42-43 \%$ of their NF cohort. OF was absent in the majority of PNAF hospitalizations in the present cohort, likely contributing to the low case fatality. These findings are similar to those reported by Endorf and colleagues [39] in the general population, finding any OF in $30.7 \%$ of hospitalizations with necrotizing soft tissue infections, though as noted, the latter study likely underestimated the rate of $\mathrm{OF}$ in their cohort. Nevertheless, PANF in the patients described in this study was associated with substantial morbidity other than $\mathrm{OF}$, as reflected by prolonged hospital length of stay and high hospital charges. It can be hypothesized that the low frequency of OF reflects the generally healthy population in the present study. The present study demonstrated that development of $\mathrm{OF}$ increased nearly threefold during study period. The frequency of chronic comorbidities rose markedly during the same period and may have increased risk of development of OF. This explanation is supported by occurrence of OF at a rate nearly twice as high among women with chronic comorbidities as compared to those with no reported chronic illness. The association of $\mathrm{OF}$ with chronic illness was also noted in the general population with NF. Psoinos et al. [6] reported an increase in development of OF among patients with NF over the past decade that was matched by marked increase in the burden of chronic comorbidities in a study of a national data set.

We found prolonged hospital length of stay among PNAF hospitalization, far exceeding the average 2.6 days for all pregnancy-related hospitalizations in the state [40]. Hospital length of stay among patients with PANF, often prolonged, has been inconsistently reported by other investigators [9-12]. The findings of this study are comparable to prior reports on NF in the general population in the US $[6,23]$. The fiscal burden of PANF has not been previously reported. The average, inflation-adjusted (2010 dollars), total hospital charges per hospitalization in this cohort make PANF the second most expensive condition in the state, topped only by respiratory failure $(\$ 103,112)[40]$ and were nearly fivefold higher than the average charge for pregnancy-related hospitalization $(\$ 21,896)$ [40]. The average hospital charges in this study population were also markedly higher than those reported in the general population with necrotizing soft tissue infections, even when adjusted for inflation [39], though the sources of higher charges among PANF hospitalization are uncertain. Although there was no statistically significant change in hospital charges over the past decade, the trend of declining charges may have resulted from increasing care efficiencies, as reflected by simultaneous downward trend of hospital length of stay, with no rise in discharges to other facilities.

The finding of hospital mortality of $2 \%$ is markedly lower than that reported in prior case series, ranging from $17 \%$ [12] to $22 \%$ [11]. Possible explanations for the difference may include improving care, as the cited reports described patients managed during 1987-1994 [11] and 1986-2000 [12]. In addition, the small number of patients described (6 [12] and 9 [11]) 
limits the precision of case fatality estimates for the general population, with the $95 \%$ CI of case fatality in these studies overlapping those in the present cohort. Moreover, the pattern of difference in case fatality between the cohort in the present and prior reports of PNAF, is similar to that noted in the general population with NF, with large (population-level) studies describing markedly lower morality rates than single/few center reports [6]. Indeed, a recent national study of necrotizing soft tissue infections by Psoinos et al. [6], hospital mortality decreased from $9 \%$ to $4.9 \%$ between 1998 and 2010, though the described population was older with prevalent chronic illness.

The results presented here should be considered in the context of several limitations. A retrospective design and use of an administrative data set with its attendant limitations affect interpretation of these results. However, the rarity of PANF precludes practical approach to capture prospectively patient-level data. In addition, the de-identified data do not allow accounting for multiple hospitalizations by the same patient during specific period, nor to directly account for specific patients transferred between acute care hospitals. However, a similar approach with the aforementioned limitations was used by other investigators of NF in the general population [6, 39]. In addition, the de-identified nature of the data did not allow linkage to preceding pregnancy-associated hospitalizations for the postpartum hospitalization group, precluding directly exploring an association of PANF with surgical interventions and other predisposing factors during delivery hospitalizations. Moreover, because time sequence cannot be established in administrative data sets, a cause and effect relationship of events cannot be directly explored even during same hospitalization. Thus, while previous case reports and case series suggest a strong association between postpartum PANF and preceding surgical procedures, the findings of the present study of the predominance of postpartum hospitalizations among the PANF cohort provide only indirect support for this association.

The accuracy of case definition of NF in the present study has been based on ICD-9-CM coding at reporting hospitals. Administrative data sets do not provide information on pathological confirmation of NF diagnosis, raising a potential of misclassification. Nevertheless, NF diagnoses were reported very sparingly (0.004\%) among pregnancy-related hospitalization in this cohort and it is unlikely that miscoding occurred systematically or incrementally over time and thus misclassification is unlikely to explain the rise in PANF incidence. In addition, the morbidity burden of PANF in the present study, as judged by rate of ICU admission and hospital length of stay is comparable to reports on NF in the general population $[6,39]$. On the other hand, one cannot exclude underestimation of PANF in this cohort. Finally, the case identification approach used here is similar to prior investigations of NF in the general population [23, 39].

Microbiology data were not reported in the majority of PANF hospitalizations in this cohort, with similar limitation noted by others [34]. Restricting the analysis to PANF hospitalizations without additional reported sites of infection, while helping to exclude data on microbial isolates in PANF patients with non-NF infections, further limits the generalization of the results. Finally, as noted earlier, administrative data preclude ascertainment of the actual source of reported microbiology isolates among PANF 
hospitalizations. These factors affect the interpretation of these findings. However, alternative approaches at a population level can be impractical.

The results of OF in a minority of PANF hospitalization may reflect underreporting and thus underestimation of the severity of illness in this cohort. However, an established broad method was used to define OF in administrative data [17]. It is therefore unlikely that OF were selectively underreported in the state population.

The use of administrative data in this study precluded access to information on the timeliness of diagnosis of PANF and to details, time course, and appropriateness of antimicrobial therapy and resuscitative interventions, all of which may vary across institutions and individual clinicians and likely have affected the observed resource utilization and outcomes. However, as noted earlier, similar constraints affect interpretation of prior studies in the general population with NF $[23,39]$. Finally, because the state of Texas does not provide tools to convert hospital charges to costs, hospital charges were reported rather than costs of care, limiting comparisons with other cost data. However, the available charge data allowed comparisons within state population.

\section{CONCLUSION}

This research provides the first population-level study to date of PANF, describing a progressive rise in its incidence and severity over the past decade. Most PANF hospitalizations in this cohort occurred in the postpartum period and required separate hospitalization post-delivery, with nearly 1 in 4 hospitalizations associated with an additional site of infection. The majority of PANF hospitalizations required care in an ICU, with common use of lifesupport interventions. PANF patients required prolonged hospitalization with hospital charges nearly fivefold higher than those for average pregnancy-related hospitalizations, making PANF among the costliest hospital diagnoses in the state. Case fatality was low, but PANF was associated with substantial residual morbidity among hospital survivors. Further studies of PANF are needed in other populations to provide further insight into this rare complication.

\section{ACKNOWLEDGMENTS}

No funding or sponsorship was received for this study. Article processing charges were funded by Texas Tech University Health Sciences Center, Odessa. All authors meet the ICMJE criteria for authorship for this manuscript, take responsibility for the integrity of the work as whole, and have given final approval for the version published. The data described in the present study were presented in part at the annual congress of the American College of Obstetrics and Gynecology, Chicago, Illinois, on April 28, 2014.

Compliance with ethics. Because a publicly available, de-identified data set was used, this study was determined to be exempt from formal review by the Texas Tech Health Sciences Center Institutional Review Board. This article does not involve any new studies with human or animal subjects performed by any of the authors.

Conflict of interest. L. Oud and P. Watkins declare no conflict of interest. 
Open Access. This article is distributed under the terms of the Creative Commons Attribution Noncommercial License which permits any noncommercial use, distribution, and reproduction in any medium, provided the original author(s) and the source are credited.

\section{REFERENCES}

1. Fernández-Pèrez ER, Salman S, Pendem S, Farmer C. Sepsis during pregnancy. Crit Care Med. 2005;33(suppl):S286-93.

2. Robinson DP, Klein SL. Pregnancy and pregnancyassociated hormones alter immune responses and disease pathogenesis. Horm Behav. 2012;62:263-71.

3. Dillen JV, Zwart J, Schuttle J, Roosmalen JV. Maternal sepsis: epidemiology, etiology and outcomes. Cur Opin Infect Dis. 2010;23:249-54.

4. Dolea C, Stein C. Global burden of maternal sepsis in the year 2000. Evidence and information for policy, World Health Organization, Geneva, July 2003. http://www.who.int/healthinfo/statistics/ bod_maternalsepsis.pdf. Accessed May 31, 2012.

5. Ward RG, Walsh MS. Necrotizing fasciitis: 10 years' experience in a district general hospital. Br J Surg. 1991;78:488-9.

6. Psoinos CM, Flahive J, Shaw JJ, et al. Contemporary trends in necrotizing soft tissue infections in the United States. Surgery. 2013;153:819-27.

7. Mills MK, Faraklas I, Davis C, Stoddard GJ, Saffle J. Outcomes from treatment of necrotizing soft tissue infections: results from the National Surgical Quality Improvement Program database. Am J Surg. 2010;200:790-7.

8. Simmonds M. Necrotizing fasciitis and group A streptococcus toxic shock-like syndrome in pregnancy: treatment with plasmapheresis and immunoglobulin. Int $\mathrm{J}$ Obstet Anesth. 1999;8:125-30.

9. Penninga L, Wettergren A. Perforated appendicitis during near-term pregnancy causing necrotizing fasciitis of the lower extremity: a rare complication of a common disease. Acta Obstet Gynecol Scand. 2006;85:1150-1.

10. Nikolau M, Zampakis P, Vervita V, et al. Necrotizing fasciitis complicating pregnancy: a case report and literature review. Case Rep Obstet Gynecol. 2014. doi:10.1155/2014/505410.

11. Goepfert AR, Guinn DA, Andrews WW, Hauth JC. Necrotizing fasciitis after cesarean section. Obstet Gynecol. 1997;89:409-12.

12. Gallup DG, Freedman MA, Megilar RV, Freedman SN, Nolan TE. Necrotizing fasciitis in gynecologic and obstetric patients: a surgical emergency. Am J Obstet Gynecol. 2002;187:305-11.

13. Aronoff DM, Mulla ZD. Postpartum invasive group A streptococcal disease in the modern era. Infect Dis Obstet Gynecol. 2008. doi:10.1155/2008/796892.

14. Texas inpatient public use data file. Texas Health Care Information Center for Health Statistics. Texas department of state health services. http://www. dshs.state.tx.us/thcic/hospitals/Inpatientpudf.shtm. Accessed Aug 2, 2013.

15. Vital statistics annual reports. Texas department of state health services. https:/www.dshs.state.tx.us/ chs/vstat/annrpts.shtm. Accessed July 28, 2013.

16. Deyo RA, Cherkin DC, Ciol MA. Adapting a clinical comorbidity index for use with ICD-9-CM administrative databases. J Clin Epidemiol. 1992;45:613-9.

17. Lagu T, Rothberg MB, Shieh M, Pekow PS, Steingrub JS, Lindenauer PK. Hospitalizations, costs and outcomes of severe sepsis in the United States 2003 to 2007. Crit Care Med. 2012;40:754-61.

18. Kuklina EV, Whieman MK, Hillis SD, et al. An enhanced method for identifying obstetric deliveries: implications for estimating maternal morbidity. Matern Child Health J. 2008;12:469-77.

19. Nybo Andersen AM, Wohlfahrt J, Christens P, Olsen J, Melbye M. Maternal age and fetal loss: population based register linkage study. BMJ. 2000;320:1708-12.

20. Ammon Avalos L, Galindo C, Li DK. A systematic review to calculate background miscarriage rates using life table analysis. Birth Defects Res A Clin Mol Teratol. 2012;94:417-23.

21. Ellis Simonsen SM, van Orman ER, Hatch BE, et al. Cellulitis incidence in a defined population. Epidemiol Infect. 2006;134:293-9.

22. Das DK, Baker MG, Venugopal K. Increasing incidence of necrotizing fasciitis in New Zealand: a nationwide study over the period 1990 to 2006 . J Infect. 2011;63:429-33.

23. Mulla ZD, Gibbs SG, Aronoff DM. Correlates of length of stay, cost of care, and mortality among 
patients hospitalized for necrotizing fasciitis. Epidemiol Infect. 2007;135:868-76.

24. Hussein QA, Anaya DA. Necrotizing soft tissue infections. In: Kumar A, editor. Life-threatening infections: part 2. Philadelphia: Elsevier. Crit Care Clin. 2013;29:795-806.

25. Magann EF, Doherty DA, Sandlin AT, Chauhan SP, Morrison JC. The effects of an increasing gradient of maternal obesity on pregnancy outcomes. Aust N Z J Obstet Gynecol. 2013;53:250-7.

26. Bautista-Castalano I, Henriquez-Sanchez P, Aleman-Perez N, Garcia-Salvador JJ, GarciaHernandez JA, Serra-Majem L. Maternal obesity in early pregnancy and risk of adverse outcomes. PLoS ONE. 2013;8:e80410. doi:10.1371/journal.pone. 0080410.

27. Weiss AJ, Elixhauser A. Obesity-related hospitalizations, 2004 versus 2009: statistical brief \#137. Healthcare Cost and Utilization Project (HCUP). Statistical briefs: agency for healthcare policy and research (US); 2006-2012. http://www. hcup-us.ahrq.gov/reports/statbriefs/sb137.jsp. Accessed Sept 9, 2013.

28. Menacker F, Hamilton BE. Recent trends in cesarean delivery in the United States: NCHS Data Brief No. 35, 2010. Center for Disease Control and Prevention. http://www.cdc.gov/nchs/data/data briefs/db35.pdf. Accessed Sept 21, 2013.

29. Schumacher H, Tehrani H, Irwin MS, Malata CM. Abdominoplasty as an adjunct to the management of peri-caesarian section necrotizing fasciitis. J Plast Reconstr Aesthet Surg. 2008;61:807-10.

30. Nissman KW, Nissman DB, Leighton BL, Varaday SS, Lockhart EM. Necrotizing fasciitis after cesarean section. Anesthesiology. 2011;115:1301.

31. de Moya MA, del Carmen MG, Allain RM, Hirschberg RE, Shepard JO, Kradin RL. Case 33-2009: a 35-year-old woman with fever, abdominal pain, and hypotension after cesarean section. N Engl J Med. 2009;361:1689-97.

32. Bernal NP, Latenser BA, Born JM, Liao J. Trends in 393 necrotizing acute soft tissue infection patients. Burns. 2012;38:252-60.

33. Widjaja AB, Tran A, Cleland H, Leung M, Millar I. The hospital costs of treating necrotizing fasciitis. ANZ J Surg. 2005;75:1059-64.

34. Walkey AJ, Wiener RS, Lindenauer PK. Utilization patterns and outcomes associated with central venous catheter in septic shock: a populationbased study. Crit Care Med. 2013;41:1450-7.

35. Tillou A, StHill CR, Brown C, Velmahos G. Necrotizing soft tissue infections: improved outcomes with modern care. Am Surg. 2004;70:841-4.

36. Das DK, Baker MG, Venugopal K. Risk factors, microbiological findings and outcomes of necrotizing fasciitis in New Zealand; a retrospective chart review. BMC Infect Dis. 2012;12:348.

37. Wunsch H, Angus DC, Harrison DA, et al. Variation in critical care services across North America and Western Europe. Crit Care Med. 2008;36:2787-93.

38. Seymour CW, Iwashyna TJ, Ehlenbach WJ, Wunsch $\mathrm{H}$, Cooke CR. Hospital-level variation in use of intensive care. Health Serv Res. 2012;47:2060-80.

39. Endorf FW, Klein MB, Mack CD, Jurkovich GJ, Rivara FP. Necrotizing soft tissue infections: differences in patients treated at burn centers and non-burn centers. J Burn Care Res. 2008;29:933-8.

40. Facts and figures: statistics on hospital-based care in Texas, 2009. Texas Health Care Information Collection. DSHS Publication \# E87-11648. http:// www.dshs.state.tx.us/thcic/publications/hospitals/ statisticalreports.shtm. Accessed Aug 25, 2013. 